\title{
A Mathematical Model of Exoprotein Production in Bacteria
}

\author{
K. D. Coleman \\ Department of Microbiology and Molecular Genetics, Harvard Medical School, \\ Boston MA 02115, U.S.A.
}

AND

\author{
A. C. Fowler
}

Department of Mathematics, Massachusetts Institute of Technology, Cambridge, MA 02139, U.S.A.†

[Received 4 November 1983 and in revised form 15 February 1984]

\begin{abstract}
We present a simple mathematical model for the synthesis of extracellular proteins by a class of bacteria which secrete significant quantities of this exoprotein in late-exponential and stationary phases. This model is the simplest generalization of Michaelis-Menten kinetics (the Monod model) and agrees well with laboratory experiments in batch culture. The model may serve as a simple prototype for the analysis of certain virulent bacterial infections in vivo, particularly that of Pseudomonas aeruginosa in burn wounds.
\end{abstract}

\section{Introduction}

BACTERIA SYNTHESIZE thousands of different types of molecules, most of which are compartmentalized within the confines of the cell envelope. Certain molecules, however, perform more useful functions outside the cell. These are often proteins with degradative or defensive activities which allow bacteria to scavenge for nutrients or compete more effectively with other bacteria in hostile environments. Man has taken advantage of these extracellular proteins in numerous industrial and medical processes including the synthesis of organic compounds, production of cheese, destruction of thrombi and emboli in the circulatory system, and for the production of vaccines active against many bacterial infections.

We are interested here in a particular class of microorganisms which show a pronounced production of exoprotein during late-exponential and early stationary phases of growth. Exoprotein formation limited to these late stages of microbial growth (in batch culture) is a distinctive feature of this class-most microorganisms produce extracellular products simultaneously with cell growth (for reviews see Priest (1977) and Glenn (1976)). Examples of microorganisms in this class are Staphylococcus aureus (Abbas-Ali \& Coleman, 1977), Bacillus subtilis (Coleman, 1967; Sippola \& Mäntälä, 1981), B. amyloliquefaciens (Coleman \& Brown, 1975), and Pseudomonas aeruginosa (Chung \& Collier, 1977).

† Address for correspondence 
We have chosen $P$. aeruginosa as the prototype organism for our studies because of the large volume of published data on growth and exoprotein formation by this organism both in vitro and in vivo during experimental infections. $P$. aeruginosa is a gram-negative rod frequently implicated in life-threatening infections of immunologically compromised patients or those with severe burns (Flick \& Cluff, 1976; Pruitt, 1974). During infection of a burn wound, small numbers of $P$. aeruginosa cells colonize and begin to multiply within the burn eschar. The high growth rate, abundance of nutrients, and absence of an intact host defence system allow the bacteria to grow almost unencumbered; normal host defence mechanisms flawlessly protect healthy individuals from infection with this organism.

At later times during infection the entire burn eschar becomes occupied by multiplying microorganisms, though at this time few organisms are able to spread to surrounding tissues (Steiritz \& Holder, 1975). Available nutrients are also beginning to become scarce and the organisms' growth rate begins to decline. At the same time, tissue-damaging exoproteins are produced which allow the colony to spread further. Some of these exoproteins are termed 'toxins' because they produce lethal effects in experimental animals when administered in low doses (Gill, 1982). The lethal outcome of many bacterial infections often relies on the ability of microorganisms to produce exotoxins in a susceptible host, where the capacity of that host to neutralize or slow production of these exotoxins is limited.

Pseudomonas burn wound sepsis is an especially disturbing problem since mortality rates have remained high despite the advent of improved antibiotic drugs and regimens (Baltch, Hammer, Smith, \& Sutphen, 1979). Other modes of treatment are currently being tested; these include the development of vaccines (Jones, 1981) and the use of therapies which potentiate or supplement the immune system (Dale, Reynolds, Pennington, Elin, Pitts, \& Graw, 1974). Whatever mode of treatment is ultimately found to be most successful, an understanding of the microbe-host interaction will be necessary. To this end we have constructed an exploratory mathematical model which describes the relationship between bacterial cell growth and exoprotein formation. This work may ultimately provide the basis for mathematical models of microbe-host interactions as they occur in disease.

There is a wealth of literature on the subject of constructing mathematical models to describe bacterial cell growth. Much of this stems from the emergence in the last fifteen years of the industrial significance of bioengineering processes, for example in the production of penicillin. Recent reviews are those of Blanch (1981) and Roels \& Kossen (1978). The great majority of models are based on the original work of Monod (1942), who proposed that the specific growth rate $X^{-1} \mathrm{~d} X / \mathrm{d} t$ of a bacterial population of a biomass $X$ (typical units are those of mass density, e.g. $\mathrm{mg} / \mathrm{ml}$.) might be given in terms of the surrounding substrate concentration $S$ by the Monod equation:

$$
\frac{1}{X} \frac{\mathrm{d} X}{\mathrm{~d} t}=\frac{\mu S}{K_{\mathrm{s}}+S}
$$


Here $S$ may be thought of as a nutrient for the cells, which enables them to multiply. The efficiency of conversion of $S$ to $X$ is measured by the yield constant, $Y$, given by:

$$
\frac{\mathrm{d} X}{\mathrm{~d} t}=-\mathrm{Y} \frac{\mathrm{d} S}{\mathrm{~d} t} .
$$

In reality, a medium might contain many 'nutrient' substances. In this case, that which is consumed first (the limiting substrate) takes the role of $S$ above. Some bacteria can thrive even in the absence of a primary nutrient, so that they switch from one Monod-type process to another when the first nutrient is consumed. For example, Saccharomyces cerevisiae exhibits such diauxic growth in a medium containing glucose and ethanol (the ethanol actually being excreted during glucose consumption) (Bijkerk \& Hall, 1977).

The relation (1.1) is the Michaelis-Menten relation for a simple catalytic enzyme reaction, and as such draws an analogy between such a reaction and the process of bacterial cell replication. Actually, there is a biochemical as well as a mathematical analogy, since the mechanism of cell reproduction does involve a large number of enzyme reactions, and so one can think mathematically of (1.1) as being a crude representation (or truncation) of the actual system under consideration. Furthermore, the Monod equation has a certain quantitative validity, and is able to reproduce observations reasonably well in both batch and continuous cultures (Herbert, Elsworth, \& Telling, 1956). It has been used as the basic building block of most subsequent models (e.g. Barford \& Hall, 1978; Blanch, 1981; Koga, Burg, \& Humphrey, 1967; Verhoff, Sundaresan, \& Tenney, 1972).

Equations (1.1) and (1.2) are easily integrated to give:

$$
X+Y S=Y S_{0}
$$

and

$$
\ln X+\delta \ln \left(\frac{X}{Y S_{0}-X}\right)=\mu t,
$$

where $S_{0}$ is a constant of integration (roughly: the initial substrate concentration), and the time origin of (1.4) is arbitrary; $\delta$ in (1.4) is given by

$$
\delta=K_{\mathrm{s}} / S_{0}
$$

and is a relative measure of the saturation nutrient density $K_{\mathrm{s}}$ compared to the initial nutrient density. Particularly if $\delta$ is quite small, two 'phases' are evident from (1.4): firstly, an exponential (often called log growth) phase, in which $X<Y S_{0}$, and

$$
\ln X \approx \mu t,
$$

i.e. the population grows exponentially in the presence of an adequate supply of nutrients; secondly, a stationary phase when $X \approx Y S_{0}$, and

$$
Y S_{0}-X \approx \text { constant } \times \exp (-\mu t / \delta) \text {, }
$$

and $X \rightarrow Y S_{0}$ as $t \rightarrow \infty$. 
Both these phases are typical of microbial growth, but there exist in addition two further phases which cannot be modelled by the simple Monod equation. These are the decline phase (at the end of the stationary phase) when cells may 'die off' by lysing, and a 'lag' phase before the exponential phase, when growth is less rapid; this may be thought of as being due to the bacteria adjusting to their nutrient environment.

In order to accommodate these and other commonly observed phenomena, a variety of more sophisticated models has been presented (Barford \& Hall 1981). Perhaps most notable (at least from the mathematical point of view) are the structured models, in which some variation in cell quality is admitted. This can take a number of forms, of which the concept of a structured biomass is the most prevalent; the cell is considered to consist of two or more constituents, each of which has a distinct function (Grenney, Bella, \& Curl, 1973; Pamment, Hall, \& Barford, 1978; Verhoff et al., 1972; Williams, 1967). Typically there is a component $A$ which is able to take up substrate and convert it into a form which can be used to promote all growth in a second component B. Bijkerk \& Hall (1977) interpret these as the (lumped) respiratory and glycolytic pathways, and although such an analogy is hardly less crude than that of the Monod model, it is a useful conceptual framework. Other cell qualities that have been considered are age distribution (Shu, 1961), and its effect on metabolic function (Brown \& Vass, 1973), although roughly speaking, one can imagine all of these as having the effect of building some sort of delayed response into the system. Such structured models are able to model the lag phase, as well as other phenomena (e.g. diauxic growth).

A further development, particularly in bioengineering studies, has been the extension of the basic Monod model to describe situations where the bacteria synthesize extracellular enzymes, for example the production of penicillin. Virtually all these models are semi-empirical, as opposed to mechanistic (Barford \& Hall, 1978), for example in the studies of Constantinides, Spencer, \& Gaden (1970). Others are self-confessed simulations, hardly aimed at anything other than curve-fitting (Heijnen \& Roels, 1979; Koga et al., 1967). At the mechanistic level, Van Dedem \& Moo-Young (1973) describe an inductor-repressor model, and Kaushik, Gondo, \& Venkatasubramanian (1979) describe catabolite repression. In both these cases, enzyme synthesis happens concurrently with bacterial growth, and also depends on it. Thus the particular class of extracellular enzymes with which we are concerned is considered to have a fundamentally different mode of production. We have not been able to find any reference in the literature to a mathematical model for this kind of enzyme synthesis.

Coleman, Brown, \& Stormonth (1975) proposed a switching mechanism which could account for the differential rates of exoprotein production during exponential, late exponential, and stationary phases of microbial growth in batch culture. Two separate effects, when superimposed, were postulated to result in the rise in exoprotein formation as cell growth slows. The first is an overabundance of ribonucleotide building blocks which are no longer required to produce additional biosynthetic machinery, and which are now available for exoprotein messenger RNA (mRNA) synthesis. The second effect involves the selective transcription of 
genes encoding exoproteins, a discriminatory function thought to reside on the large RNA polymerase complex. The end result would be a switch from cellular to extracellular protein synthesis. To this day the Coleman et al. proposal remains an attractive and unrivalled model to explain the biochemical basis of this cellular switch.

\section{Mathematical model}

The constituents of a simple, unstructured mathematical model for the process described in Section 1 are the bacterial biomass concentration $X$, the (limiting) substrate (nutrient) density $S$, and the extracellular product concentration $P$. The simplest analogous 'reaction' scheme (see Verhoff et al., 1972; Bijkerk \& Hall, 1977) to describe this process is

$$
X+a_{1} S \stackrel{\backslash S \downarrow}{\longrightarrow} X+a_{2} P,
$$

where we have indicated that the 'reaction' switches from one mode to another as $S$ decreases. By considering as a first option the simplest unstructured model for extracellular protein synthesis, we implicitly focus attention on the (exponential) growth phase, and the subsequent (stationary phase) product formation. We are thus avoiding a discussion of the lag phase, polyauxic growth, cell structure, etc.

The upper (growth) phase of the schematic 'reaction' (2.1) is commonly represented by the law of mass action,

$$
\frac{\mathrm{d} X}{\mathrm{~d} t}=k_{1}\left(\frac{S}{K_{\mathrm{s}}+S}\right) X
$$

as described in $\$ 1$. The saturating rate constant $r=k_{1} S /\left(K_{\mathrm{s}}+S\right)$ is the Monod term; the precise form of $r(S)$ is open to discussion (e.g. Edwards, 1970). The Michaelis constant $K_{\mathrm{s}}$ is a 'saturation level' of nutrient which will roughly describe where the switching occurs.

In order to model the proposed 'switch' (Coleman et al., 1975) between growth and production, we have essentially two possibilities. One is to construct a feasible (and convoluted) 'reaction scheme' whose purpose is to represent the switching process. Since the biochemical understanding of this process is still controversial, this may not be worthwhile, and in any case is not in keeping with the use of the simplistic Monod model. A second possibility is to augment the Monod model in a macroscopically self-consistent manner, which (like the Monod model) is not precisely defined chemically; it is this approach we adopt here.

Since the switch is considered to depend on the nutrient level, we might suppose that as the nutrient level decreases, the fraction of total cell activity devoted to exoenzyme production gradually increases, whereas that contributing to cell growth correspondingly decreases. The precise mode of change may not be relevant since any discussion of cell population characteristics represents an average over many different cells. One particular choice is then to stipulate that a cell switches abruptly from growth mode to production mode at, for example, 
zero nutrient intake. We use this formal idealization of the switching, together with a discussion of the averaging process, to obtain a description of exoenzyme production.

Let us suppose that the specific growth rate is still given by (2.2). We now interpret what the equation (2.2) means from the point of view of the individual microorganisms. As with all models like (2.2) which involve differential equations, the assumption is implicitly made that the aggregate of bacteria can be treated as a continuum whose size depends continuously (and differentiably) on time. This is an idealization which incorporates the fact that the individual microorganisms are very small (or very numerous). In some sense the resulting differential equations we consider represent local averages of the stated quantities. Thus the equation (2.2) can be taken to represent the spatially averaged growth behaviour of a large colony of bacteria. Now from the point of view of an individual microorganism, it is reasonable to suppose that in the presence of limitless quantities of nutrient, each organism grows at a rate $k_{1}$ (that is, its doubling time is $\left(1 / k_{1}\right) \ln 2$ ). One way of viewing (2.2) is then that when the nutrient level is $S$, this is only sufficient for $S /\left(K_{\mathrm{s}}+S\right)$ of the bacteria to grow at their full rate: these use all the nutrients up (they are just saturated) whereas the other $K_{\mathrm{s}} /\left(K_{\mathrm{s}}+S\right)$ get no nutrient at all. Thus we conceptually think of (2.2) as describing the spatially averaged behaviour of bacteria which are either starving or saturated. It should be emphasised that this is purely a conceptual interpretation, but it is equivalent when averaged to a more realistic interpretation (i.e. that the bacteria simply grow more slowly because of the limited nutrient supply).

Although not a difficult point to grasp, we have laboured the argument somewhat, since for the particular case of a 'bimodal' microorganism with two modes of operation, it has an important consequence, which is as follows. Let us suppose that such a microorganism is enveloped in a subsaturation level of nutrient $\left(S \leq K_{\mathrm{s}}\right)$. As before we can consider a small space volume of bacteria to be composed of those which have sufficient nutrient to multiply at their maximal rate (this is a fraction $S /\left(K_{\mathrm{s}}+S\right)$ of the total density); the remainder have zero nutrient available to them. By virtue of the bimodal assumption, these latter do not grow, but they produce extracellular enzymes at a constant rate $k_{2}$ per unit biomass. Since a fraction $K_{\mathrm{s}} /\left(K_{\mathrm{s}}+S\right)$ have no nutrient available it follows that the average rate of protease production must be

$$
\frac{\mathrm{d} P}{\mathrm{~d} t}=k_{2}\left(\frac{K_{\mathrm{s}}}{K_{\mathrm{s}}+S}\right) X
$$

we emphasise that these conceptual assumptions are only a means to the end of understanding the equations (2.2) and (2.3): we do not claim that any such precise 'switch' as described above actually occurs, but only that a logical conclusion from prescribing a Michaelis-Menten specific growth rate (2.3) is that we must also have a nutrient-dependent product formation rate which varies in the opposite manner.

Of course, the above empirical reasoning is not very satisfactory, and a more mechanistic hypothesis might be preferred. In that case, one firstly idealizes the Monod growth mechanism in terms of an intermediary 'complex', analogous to 
the simple Michaelis-Menten enzyme kinetics. This can be done by visualizing the bacterium as a cell with a large number of receptors, which bind reversibly to the (limiting) substrate, and which form (ultimately) biomass when bound, and also (in the case here) exoprotein when not bound. Suppose that at time $t$, a fraction of sites $R(t)$ is bound to substrate. If the mean time of reaction (of biomass formation) is $\tau$, and the mean collision time of substrate with biomass is $\theta$, then the equation for $R$ is

$$
\frac{\mathrm{d} R}{\mathrm{~d} t}=\frac{R}{\tau}-\frac{(1-R)}{\theta}
$$

analogously to the pseudo-steady state hypothesis, we suppose $\theta, \tau$ are so small that the reaction (2.3a) is essentially in equilibrium: there follows

$$
R=\frac{\tau}{\theta+\tau}
$$

The derivation of this relation is precisely analogous to that of the Langmuir adsorption isotherm (Szekely, Evans, \& Sohn, 1976). If one now realizes that $\theta$ will vary inversely with $S$, e.g. $\theta \propto 1 / S$, then $(2.3 b)$ is $R=S /(\rho+S)$, for some constant $\rho$. Additionally, one would have $\mathrm{d} X / \mathrm{d} t \propto R, \mathrm{~d} P / \mathrm{d} t \propto 1-R$, which are precisely the equations (2.2) and (2.3). The advantage of this latter discussion is that it constitutes a more specific mechanistic picture; the disadvantage may be that it seems to imply a particular biochemical function for the cell, which is liable to be inaccurate.

Equations (2.2) and (2.3) constitute the basis of our mathematical model. They are supplemented by an equation describing decrease of the limiting nutrient, obtained from (2.1) and the additional 'reaction'

$$
\text { Proto- } S+P \rightarrow P+S,
$$

which describes the action of extracellular protease in degrading 'proto-nutrient' (limitlessly available) into assimilable material for the cell. This was suggested by Cicmanec \& Holder (1979) as an important feature of Pseudomonas virulence in burned mice.

With the constitutive assumptions (2.2) and (2.3) relating the switch, the model (2.1) and (2.4) is therefore represented by the equations:

$$
\begin{aligned}
& \frac{\mathrm{d} X}{\mathrm{~d} t}=k_{1}\left(\frac{S}{K_{\mathrm{s}}+S}\right) X, \\
& \frac{\mathrm{d} P}{\mathrm{~d} t}=k_{2}\left(\frac{K_{\mathrm{s}}}{K_{\mathrm{s}}+S}\right) X,
\end{aligned}
$$

and

$$
\frac{\mathrm{d} S}{\mathrm{~d} t}=k_{3} P-\frac{k_{1}}{Y}\left(\frac{S}{K_{\mathrm{s}}+S}\right) X,
$$

where $Y$ is the yield constant, and $k_{1}, k_{2}$, and $k_{3}$ are rate constants. We have neglected any self-degradation of proteins as being small. 
These equations are supplemented by initial conditions:

$$
X=X_{0}, S=S_{0}, \quad \text { and } P=0 \text { at } t=0,
$$

and represent conditions in a batch culture. If $k_{2}=0$, they reduce to the Monod model. If $k_{2} \neq 0$, they are non-linear and not obviously tractable, although if $k_{3}=0$, they are easily solved by quadrature of (2.6), using (1.3) and (1.4). The result is

$$
P=\frac{k_{2} Y K_{\mathrm{s}}}{k_{1}} \ln \left(\frac{Y S_{0}-X_{0}}{Y S_{0}-X}\right)
$$

With $k_{3} \neq 0$ in (2.7), a solution is not so straightforward, although it can be readily seen from (2.5) and (2.6) that the solutions are capable of simulating both exponential growth $\left(S \gg K_{\mathrm{s}}\right)$ and a stationary phase linear production phase $\left(S \ll K_{\mathrm{s}}\right)$, as is experimentally realized (Coleman, 1967).

\section{Dimensionless model}

In order to proceed further, we construct dimensionless equivalents of the equations. To do so, we choose scales based on the solution of (2.5)-(2.7) in the case $k_{3}=0$. This solution-partially given in (2.9)-is:

$$
\begin{gathered}
(1+\delta) \ln X-\delta \ln \left(Y S^{*}-X\right)=k_{1}\left(t-t_{0}\right), \\
X=Y\left(S^{*}-S\right),
\end{gathered}
$$

and

$$
P=\frac{k_{2} K_{\mathrm{s}} Y}{k_{1}} \ln \left(\frac{\tilde{S}}{S}\right)
$$

where the three constants $t_{0}, S^{*}$, and $\tilde{S}$ are chosen to satisfy the initial conditions, say (2.8); for example, $t_{0}=0, \tilde{S} \approx S^{*}$ is approximately the initial substrate concentration $S_{0}$. Additionally,

$$
\delta=K_{\mathrm{s}} / S^{*}
$$

We assume $\delta \leqslant 1$, and write:

$$
t=\tau / k_{1}, \quad S=S^{*} s, \quad X=Y S^{*} x, \quad \text { and } P=\left[k_{2} Y S^{*} / k_{1}\right] p,
$$

the last from (2.6), when $X \rightarrow Y S^{*}, S \rightarrow 0$. The dimensionless equations can thus be written in the form:

$$
\begin{aligned}
& \dot{x}=[1+\delta / s]^{-1} x, \\
& \dot{p}=[1+s / \delta]^{-1} x,
\end{aligned}
$$

and

$$
\dot{s}=\alpha p-[1+\delta / s]^{-1} x,
$$

where $\delta$ is defined by (3.2), $\dot{x} \equiv \mathrm{d} x / \mathrm{d} \tau$, and

$$
\alpha=k_{3} k_{2} Y / k_{1}^{2} \text {. }
$$


The solution depends on the two dimensionless parameters $\alpha$ and $\delta$, and in particular the simple solution (3.1) is predicated on the basis of the limit $\alpha \rightarrow 0$, i.e. $\alpha$ is 'small': thus we need to obtain estimates of $\alpha$ and $\delta$ to justify or invalidate the use of such an approximation.

The parameters $k_{1}, k_{2}, k_{3}, Y, K_{\mathrm{s}}$, and $S^{*}$ are estimated as follows.

(a) $k_{1}$ is the growth rate in the exponential phase, and typical quoted values of doubling times for Pseudomonas aeruginosa (Cicmanec \& Holder, 1979), Staphylococcus aureus (Coleman \& Abbas-Ali, 1977) and Bacillus subtilis (Coleman, 1967) are in the range 0.5 hour to 3 hour. We thus take

$$
k_{1} \sim 1 \text { hour }^{-1} \text {. }
$$

(b) $k_{2}$ is the rate of extracellular protein synthesis in starvation conditions. A number of different experiments for different organisms yield values in the range $0.025-0.25 \mathrm{mg} \mathrm{ml}^{-1} \mathrm{hr}^{-1}$ at bacterial dry weights of about 1 to $6 \mathrm{mg} \mathrm{ml}^{-1}$ (AbbasAli \& Coleman, 1977; Coleman 1967, 1981; Coleman, Jakeman \& Martin, 1978; Robinson, Keating, \& Sloan, 1980; Williams \& Wimpenny, 1977). We take

$$
k_{2} \sim 0.01 \mathrm{mg} \text { (protease) } \mathrm{mg} \text { (bacteria) }{ }^{-1} \mathrm{hr}^{-1} \text {. }
$$

(c) $k_{3}$ is the rate of production of nutrient by protease. Typical enzyme activities, e.g. Wheatley \& Moo-Young (1977), suggest a value of about

$$
\left.k_{3} \sim 10^{3} \mathrm{mg} \text { (nutrient) } \mathrm{mg} \text { (protease) }\right)^{-1} \mathrm{hr}^{-1} \text {. }
$$

(d) The yield constant, $Y$, is generally (Herbert et al., 1956) of order one, let us say

$$
Y \sim 0.5 \mathrm{mg} \text { (bacteria) } \mathrm{mg} \text { (substrate) })^{-1}
$$

(but see also later discussion).

(e) The Michaelis constant $K_{\mathrm{s}}$ depends on both the organism, and on which nutrient is rate-limiting. Typical values are given by Brock $(1974): \sim 2 \times 10^{-5} \mathrm{M}$ (molar) for glucose ( $E$. coli), $\sim 10^{-6} \mathrm{M}$ for oxygen (yeast), $\sim 3 \times 10^{-8} \mathrm{M}$ for phosphate (spirillum). The oxygen value corresponds to $4 \times 10^{-5} \mathrm{mg} \mathrm{ml}^{-1}$. Herbert et al. (1956) quote higher values of order $10^{-3} \mathrm{mg} \mathrm{ml}^{-1}$ for carbohydrate substrates. For Pseudomonas, Nyberg and Clarke (1978) found nitrogen limitation occurring at $1 \mathrm{mg} \mathrm{ml}^{-1}$ in a succinate/histidine medium. Button (1978) comments that other limiting mechanisms may lower the apparent value of limiting substrate concentration below its actual saturation level: we will have a further discussion of this in Section 5. With some reservation, we consider a general 'typical' value of

$$
K_{\mathrm{s}} \sim 10^{-3} \mathrm{mg} \mathrm{ml}^{-1}
$$

(e.g. glucose limited in E. coli cultures).

(f) The 'initial' nutrient level $S^{*}$ is entirely dependent on the particular experiment. In many experiments, the medium is enriched with nutrient (e.g. Coleman \& Abbas-Ali, 1977) at a typical level of $10 \mathrm{mg} \mathrm{ml}^{-1}(\sim 1 \%$ weight per volume). Cicmanec and Holder (1979) using burnt-skin extract had an initial protein concentration of $10^{-1} \mathrm{mg} \mathrm{ml}^{-1}$. In such circumstances, aerobic bacteria growth may in fact be oxygen-limited. Water at atmospheric pressure contains 
$\sim 6 \times 10^{-3} \mathrm{mg} \mathrm{ml}^{-1}$ dissolved oxygen, and although equilibration with the atmosphere occurs rapidly (e-folding time of 4 minutes for a three centimetre deep layer) (Dorsey, 1940), this may become ineffective as the bacterial mass increases. For the moment, and again with reservations, we take

$$
S^{*} \sim 10^{-2} \mathrm{mg} \mathrm{ml}^{-1} \text {. }
$$

With these values of the above parameters, we calculate $\alpha$ and $\delta$, and find

$$
\alpha \approx 5, \quad \delta \approx 0 \cdot 1 \text {; }
$$

we emphasise that these may be plausible orders of magnitudes, in any particular situation, the precise values can vary. Generally, (3.12) seems to indicate that nutrient production by proteases can be important (Cicmanec \& Holder 1979). Furthermore, insofar as $S$ refers to only one (rate-limiting) medium, the effective yield constant $Y$ used in (3.5) may well be higher. Particularly, experimental results already cited tend to have a final bacterial density of $\sim 1-10 \mathrm{mg}$ (dry weight) $\mathrm{ml}^{-1}$, which would imply that $Y$ should satisfy

$$
Y S^{*} \approx 10 \mathrm{mg} \mathrm{ml}^{-1} \text {, }
$$

which is not consistent with the values quoted above, (3.9) and (3.11). If we accept $S^{*}$ from (3.11) and $Y$ from (3.13), we should find $\alpha \sim 10^{4}$; if we accept $Y$ from (3.9) and $S^{*}$ from (3.13), we should find $\delta \sim 10^{-4}$. Thus either

$$
\alpha \sim 1, \quad \delta \ll 1
$$

or

$$
\alpha \gg 1, \quad \delta \sim 1
$$

suggests itself as a plausible state of affairs: in either case, $\alpha / \delta \gg 1$.

\section{Analysis}

We now turn to an analysis of the equations (3.4), together with initial conditions

$$
s=s_{0} \approx 1, \quad x=1-s_{0}, \quad p=0 \text { at } \quad \tau=\tau_{0} .
$$

The exact solution (3.1) when $\alpha=0\left(k_{3}=0\right)$ can be written in dimensionless form as (with $\tau_{0}$ chosen appropriately)

$$
\begin{gathered}
x+s=1, \\
(1+\delta) \ln x-\delta \ln (1-x)=\tau,
\end{gathered}
$$

and

$$
p=\delta \ln (\bar{s} / s), \quad \bar{s} \sim O(1),
$$

and we would expect the solutions to behave similarly for small $\alpha$. A typical solution is shown in Fig. 1. There is a clear exponential growth phase, in which exoprotein is essentially absent, and a subsequent stationary phase, when exoprotein increases linearly with time. This may be compared with similar figures in the experimental literature, for example Coleman (1967). 


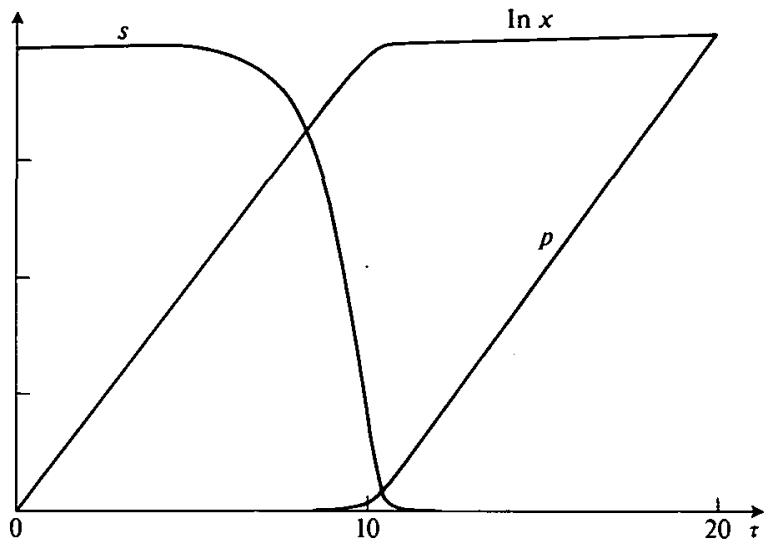

FIG. 1. Solution of (3.4) with $\delta=0 \cdot 1, \alpha=0 \cdot 001, x_{0}=10^{-4}$. In this and subsequent figures, the scales for $\ln x, p$, and $s$ have been normalized.

The easiest way to see this structure in (4.2) is when $\delta$ is small. Then, for $|\tau| \sim O(1),(4.2)$ is approximately

$$
\ln x \sim \tau
$$

and

$$
p \sim O(\delta)
$$

but when $|\tau|$ is small, $(\sim O(\delta))$, then

$$
\begin{gathered}
x \sim 1-\mathrm{e}^{-\tau / \delta}, \\
s \sim \mathrm{e}^{-\tau / \delta},
\end{gathered}
$$

and

$$
p \sim \tau,
$$

corresponding to the stationary phase exoprotein production phase. These assumptions can also be derived from the equations directly, by asymptotic solution.

Notice that if $\delta$ is small, the transition from exponential to stationary is very sharp. Also, if $\alpha$ is small but non-zero, then $s$ cannot approach zero, and no true stationary phase will occur. Both of these observations have some bearing on the realizability of the model results, and we discuss them further in Section 5 .

If $\alpha \sim 1$, but still $\delta \ll 1$, a direct asymptotic solution can still be obtained. For $\tau<0$, we have

$$
\begin{gathered}
\dot{x} \sim x, \\
\dot{p} \sim O(\delta), \text { so } \quad p \sim O(\delta),
\end{gathered}
$$

and

$$
\dot{s} \sim-x,
$$

so (4.3) still obtains. This is invalid when $s \sim O(\delta)$; then put

$$
\tau=\delta t, \quad x=1-\delta \tilde{x}, \quad s=\delta \tilde{s}, \quad \text { and } p=\delta \tilde{p},
$$


so that

$$
\begin{gathered}
\tilde{x}^{\prime}=-\frac{\tilde{s}}{\tilde{s}+1}, \\
\tilde{p}^{\prime}=\frac{1}{\tilde{s}+1},
\end{gathered}
$$

and

$$
\tilde{s}^{\prime}=\delta \alpha \tilde{p}-\frac{\tilde{s}}{\tilde{s}+1}
$$

We neglect $O(\delta)$ in $(4.7)_{3}$, and the solution is the familiar Michaelis-Menten one:

$$
\tilde{s}+\ln \tilde{s}=-t+\text { constant }
$$

and

$$
\tilde{p}=t-\tilde{s}+\text { constant }
$$

For large $t$, this behaves like (4.4), but for large $\tau(\sim O(1 / \delta)), p \sim O(1), s$ grows again, and thus $x$ starts to grow again after an intermediary lapse.

It is more direct to pick the obvious distinguished limit,

$$
\alpha=\tilde{\alpha} / \delta, \quad \tilde{\alpha} \sim O(1),
$$

and define

$$
p=\delta \tilde{p}
$$

The equations (3.4) are then

$$
\begin{aligned}
& \dot{x}=[s /(s+\delta)] x, \\
& \dot{\tilde{p}}=[1 /(s+\delta)] x,
\end{aligned}
$$

and

$$
\dot{s}=\tilde{\alpha} \tilde{p}-[s /(s+\delta)] x .
$$

For $\tilde{\alpha} \sim 1, \delta \ll 1$, and $|\tau| \sim O(1)$, we have

$$
\begin{gathered}
\dot{x} \sim x, \\
\dot{\tilde{p}} \sim x / s,
\end{gathered}
$$

and

$$
\dot{s} \sim \bar{\alpha} \bar{p}-x,
$$

whence

$$
\ln x \sim \tau,
$$

and $s$ satisfies

$$
\ddot{s}+[1-\tilde{\alpha} / s] \mathrm{e}^{\tau}=0 .
$$

From this we deduce $s$ will not go through zero (for then $\frac{1}{2} \dot{s}^{2} \sim \beta \ln |s|$ ). To find the long-time behaviour of $s$, put

$$
\theta=\mathrm{e}^{\tau} / 2
$$


so that

$$
s^{\prime \prime}+\frac{1}{\theta} s^{\prime}+4(1-\tilde{\alpha} / s)=0
$$

As $\theta$ (and $\tau$ ) $\rightarrow \infty, s$ behaves almost conservatively:

$$
\frac{1}{2} s^{2}+V(s)=E
$$

where $E$ is approximately constant, and

$$
V(s)=4(s-\tilde{\alpha} \ln |s|),
$$

with a minimum at $s=\tilde{\alpha}, V_{\min }=4 \tilde{\alpha}[1-\ln |\tilde{\alpha}|]$. For $E>V_{\min }, s$ oscillates around $\tilde{\alpha}$. In fact, $E$ will vary slowly with $\theta$, since (4.16) can be written as

$$
\frac{\mathrm{d} E}{\mathrm{~d} \theta}=-\mathrm{s}^{2} / \theta \text {. }
$$

For large $\theta, E$ is given approximately by the method of averaging:

$$
\frac{\mathrm{d} E}{\mathrm{~d} \theta} \approx-\overline{s^{\prime 2}} / \theta,
$$

where $s^{\prime 2}$ is the average value of $s^{\prime 2}$ over a period of the underlying oscillation. This can be written in the form

$$
\theta \frac{\mathrm{d} E}{\mathrm{~d} \theta} \approx 2 \frac{\int_{s^{-}}^{s^{+}}[E-V(s)]^{1 / 2} \mathrm{~d} s}{\int_{s^{-}}^{s^{+}}[E-V(s)]^{-1 / 2} \mathrm{~d} s},
$$

where $s^{+}$and $s^{-}$are the upper and lower zeros of $E-V(s)$, respectively. Evidently, $E$ approaches $V_{\min }$ as $\theta$ increases. Thus $s$ approaches $\tilde{\alpha}$ oscillatorily, and as $\theta \rightarrow \infty$ (since (4.16) is a zero-order Bessel's equation for $s \approx \tilde{\alpha}$ ). In fact

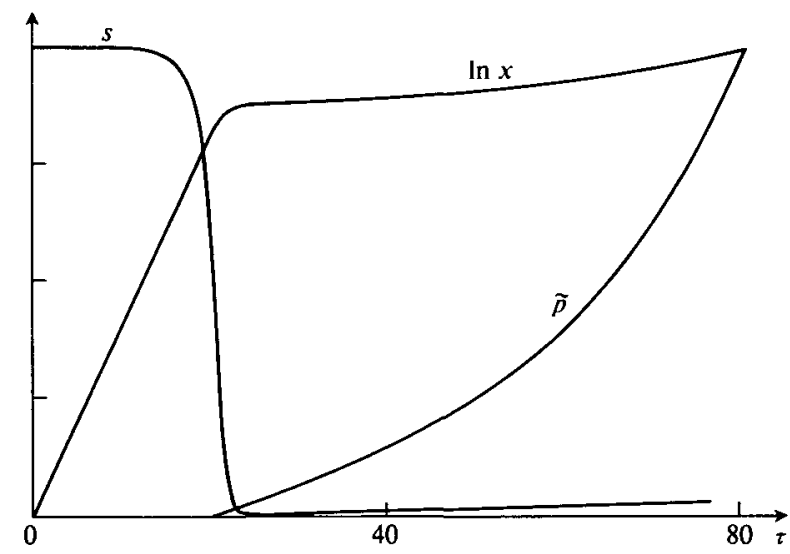

FIG. 2. Solution of (4.11) with $\delta=0.5, \tilde{\alpha}=0.001, x_{0}=10^{-6}$. 


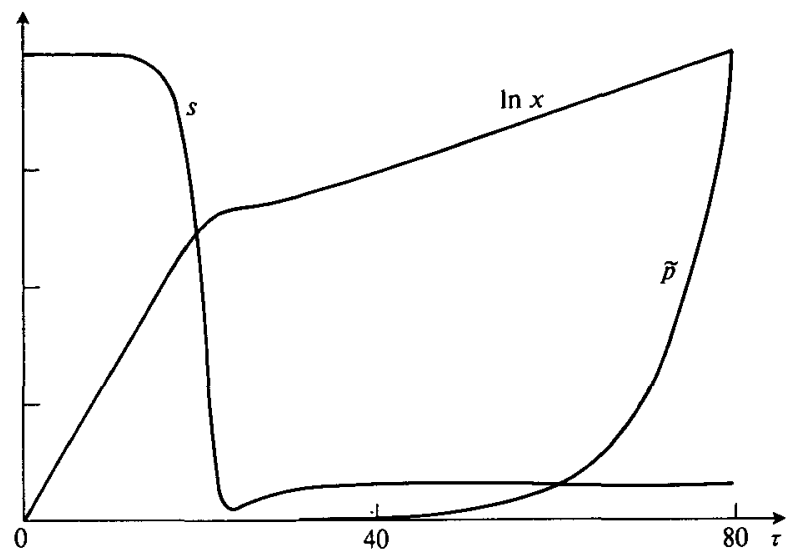

FIG. 3. Solution of (4.11) with $\delta=0.5, \tilde{\alpha}=0.01, x_{0}=10^{-6}$.

$s-\tilde{\alpha} \sim \theta^{-1 / 2} \cos [2 \theta+\phi] \sim \mathrm{e}^{-\tau / 2} \cos \left[\mathrm{e}^{\tau}+\phi\right]$. If $\tilde{\alpha} \gg \delta$, then $x$ continues to increase exponentially, being fed by the nutrient, produced by the exoprotein.

For large $\tilde{\alpha},(4.11)_{3}$ suggests that rapid equilibration occurs, so that (assuming $s \sim 1, \delta \ll 1)$

$$
\bar{p} \approx x / \bar{\alpha}
$$

(at $\tau \sim 1 / \tilde{\alpha}$ after switch-on). Then (4.11) 1,2 imply

$$
s \approx \tilde{\alpha}, \quad \ln x \sim \tau,
$$

which is as before. Figures 2 to 5 show numerically computed solutions of the equations, which exhibit the kinds of behaviour explained above. For larger $\delta$, we can expect similar kinds of solution, but these must be obtained numerically (for $\delta \sim O(1))$.

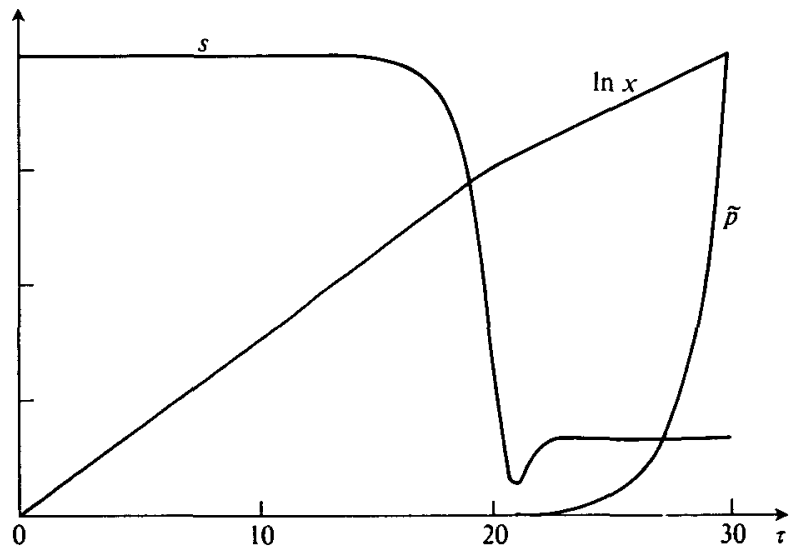

FIG. 4. Solution of (4.11) with $\delta=0 \cdot 1, \tilde{\alpha}=0 \cdot 1, x_{0}=10^{-8}$. 


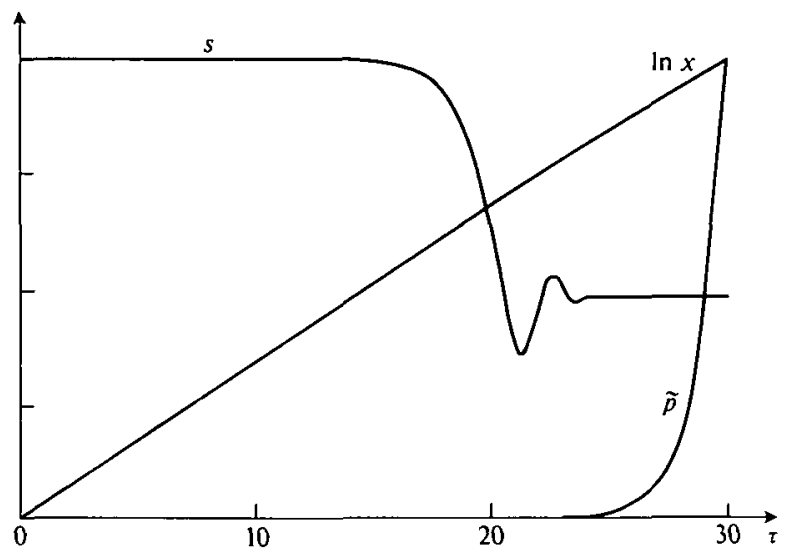

FIG. 5. Solution of (4.11) with $\delta=0.1, \bar{\alpha}=0.4, x_{0}=10^{-8}$.

In summary, we find the following class of behaviour. For $\tilde{\alpha}=\alpha \delta$ small, we see an exponential growth phase followed by a stationary phase, in which latter exoprotein is produced, and grows linearly with time. For larger $\tilde{\alpha}$, the exponential phase is followed by a slower exponential phase, where exoprotein is produced, and the bacterial growth is maintained by nutrient 'produced' by the exoprotein. At very high $\tilde{\alpha}$, this second phase occurs very quickly, and no break would be evident in the growth curves. This sequence of behaviours is portrayed schematically in Fig. 6 . The onset of significant secondary growth occurs when $\tilde{\alpha} \geq \delta$, that is $\alpha \geq 1$.

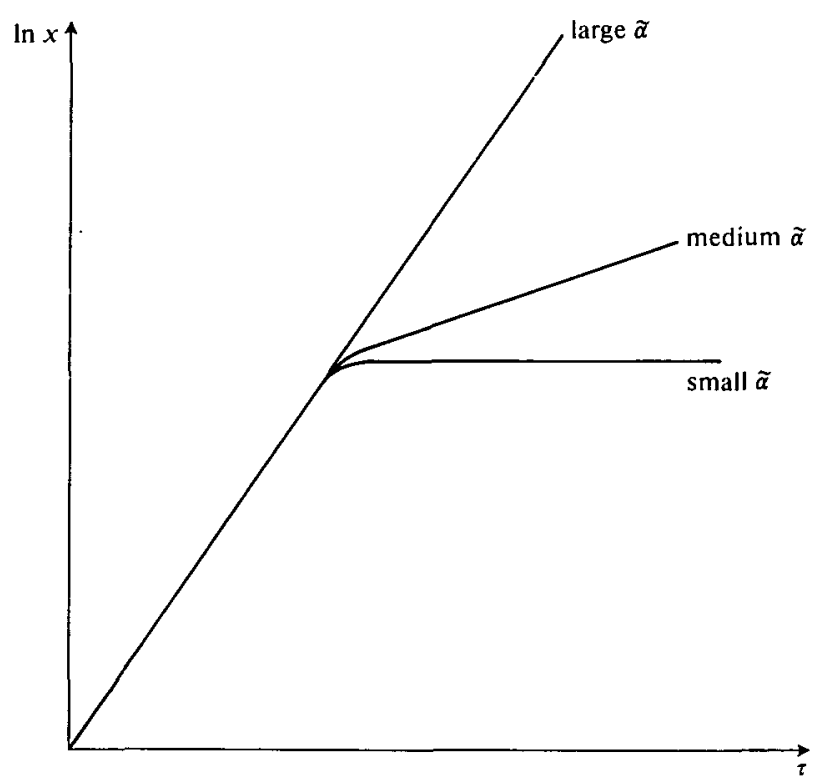

FIG. 6. Schematic growth curves for $x$ at small, intermediate, and large values of $\tilde{\alpha}$. 


\section{Discussion}

The (exact) solution given in Section 4 for $\alpha$ very small (and $\delta$ reasonably small) imitates very well the results obtained in the laboratory, particularly by Coleman (1967). There is an exponential phase, in which bacterial density increases exponentially, and exoprotein is virtually absent; this is followed by a (nearly) stationary phase in which exoprotein increases linearly with time. Furthermore, examination of (4.2) shows that exoprotein $(p)$ and bacterial density $(x)$ are related through both phases by the relation

$$
p=\delta \ln [\bar{s} /(1-x)]
$$

This compares very well with $p$ (proteinase)- $x$ (cell density) plots in Coleman (1967), and can be compared to similar plots in other work (Coleman, 1981; Robinson et al., 1980; Abbas-Ali and Coleman 1977).

This argument suggests that the simple model presented here can serve as a useful representation of the extracellular production of protein, for that class of organisms which has the switching behaviour described in the introduction. If this is so, then the subsequent analysis of Section 4 also implies that $\alpha$ is generally very small in these experiments, and that $\delta$ is reasonably (but not extremely) small, $\delta \leqslant 1$. If $\alpha$ is larger, then the analysis indicates that a transition between two phases will occur, but that the bacteria continue to grow (exponentially) in the second phase (assuming $\delta \leqslant 1$ ).

In practice, it is rather difficult to give good values of either $\alpha$ or $\delta$. The estimates in Section 3 can be expected to be unreliable, and particularly the value of $\alpha$ in (3.12) seems to be an overestimate. This is plausibly explained by uncertainties in the production rate $k_{3}$, and in interpreting what the yield constant $Y$ ought to be. It would seem that $\delta$, given by (3.2), would be much more easily identifiable, since values of $K_{\mathrm{s}}$ are reasonably well documented. However, in laboratory experiments (e.g. Coleman, 1967), the bacteria are typically grown in a nutrient-rich broth, so that it is not even obvious just what limits their growth. Furthermore, Button $(1978,1983)$ points out that apparent Michaelis constants measured in steady state conditions may underestimate the appropriate dynamic constants by orders of magnitude. One obvious candidate for limiting growth of aerobic bacteria in flask cultures is simply the availability of oxygen. We have not examined this in detail, but let us point out the work of Johnson (1967), which finds different apparent Michaelis constants for oxygen limited growth in steady and time-dependent conditions. Apart from all these concerns, it seems plausible that a more realistic structured biomass model could lead to a different effective $\delta$ than that given by (3.2).

What we are suggesting is that, although $\alpha$ and $\delta$ may not be realistically (or easily) obtainable from raw data, nevertheless, the model is still useful, if the effective values of these parameters are chosen appropriately (in conformation with the laboratory data). In that case, this model may provide the basis for a dynamic model of in vivo infection by Pseudomonas, in which a possible virulence due to protease activity (Cicmanec \& Holder, 1979) is taken into account. 


\section{REFERENCES}

AbBas-Ali, B. \& G. COLEman 1977 The characteristics of extracellular protein secretion by Staphylococcus aureus (Wood 46) and their relationship to the regulation of $\alpha$-toxin formation. J. gen. Microbiol. 99, 277-282.

Baltch, A. L., Hammer, M., Smith, R., \& Sutphen, N. 1979 Pseudomonas aeruginosa bacteremia: susceptibility of 100 blood culture isolates to seven antimicrobial agents and its clinical significance. J. Lab. clin. Med. 94, 201-214.

BARFORD, J. P. \& HAII, R. J. 1978 An evaluation of the approaches to the mathematical modelling of microbial growth. Process Biochem. 13, 22-29.

BARford, J. P. \& Hall, R. J. 1981 A mathematical model for the aerobic growth of Saccharomyces cerevisiae with a saturated respiratory capacity. Biotechnol. Bioengng. 23, 1735-1762.

Bukerk, A. H. E. \& Hall, R. J. 1977 A mechanistic model of the aerobic growth of Saccharomyces cerevisiae. Biotechnol. Bioengng. 19, 267-296.

Blanch, H. W. 1981 Microbial growth kinetics. Chem. eng. Commun. 8, 181-211.

Brock, T. D. 1974 Biology of Microorganisms. Englewood Cliffs, N.J.: Prentice-Hall.

Brown, D. E. \& VAss, R. C. 1973 Maturity and product formation in cultures of microorganisms. Biotechnol. Bioengng. 15, 321-330.

Button, D. K. 1978 On the theory of control of microbial growth kinetics by limiting nutrient concentrations. Deep-sea Res. 25, 1163-1177.

Button, D. K. 1983 Differences between the kinetics of nutrient uptake by microorganisms, growth and enzyme kinetics. Trends biochem. Sci. 8, 121-124.

Chung, D. W. \& Collier, R. J. 1977 Enzymatically active peptides from the adenosine diphoshate-ribosylating toxin of Pseudomonas aeruginosa. Infect. Immun. 16, 832841.

Cicmanec, J. F. \& Holder, I. A. 1979 Growth of Pseudomonas aeruginosa in normal and burned skin extract: role of extra-cellular proteases. Infect. Immun. 25, 477-483.

Coleman, G. 1967 Studies on the regulation of extracellular enzyme formation in Bacillus subtilis. J. gen. Microbiol. 49, 421-431.

Coleman, G. 1981 Pleiotropic compensation in the regulation of extracellular protein formation by a low $\alpha$-toxin-producing variant of Staphylococcus aureus (Wood 46). J. gen. Microbiol. 122, 11-15.

Coleman, G. \& Abbas-Ali, B. 1977 Comparison of the patterns of increase in $\alpha$-toxin and total extracellular protein by Staphylococcus aureus (Wood 46) grown in media supporting widely differing growth characteristics. Infect. Immun. 17, 278-281.

Coleman, G. \& Brown, S. 1975 Relationship between exoprotease secretion and the synthesis of ribonucleic acid and protein in Bacillus amyloliquefaciens. Antimicrob. Agents Chemotherapy 7, 840-844.

Coleman, G., Brown, S., \& Stormonth, D. A. 1975 A model for the regulation of bacterial extracellular enzyme and toxin biosynthesis. $J$. theor. Biol. 52, 143-148.

Coleman, G., Jakeman, C. M. \& Martin, N. 1978 Patterns of total extracellular protein secretion by a number of clinically isolated strains of Staphylococcus aureus. J. Gen. Microbiol. 107, 189-192.

Constantinides, A., Spencer, J. L., \& Gaden, E. L. 1970 Optimisation of batch fermentation processes I: Development of mathematical models for batch penicillin fermentations. Biotechnol. Bioengng. 12, 803-830.

Dale, D. C., Reynolds, H. Y., Pennington, J. E., Elin, R. J., Pitts, T. W., \& Graw, R. G. 1974 Experimental pneumonia due to Pseudomonas in dogs: controlled trial of granulocyte transfusion therapy. J. infec. Dis. 130, Suppl. S143-144.

Dorsey, N. E. 1940 Properties of Ordinary Water-substance. New York: Reinhold.

EDwards, V. H. 1970 The influence of high substrate concentrations on microbial kinetics. Biotechnol. Bioengng. 12, 679-712.

Fuick, M. R. \& Cluff, L. E. 1976 Pseudomonas bacteremia: review of 108 cases. Amer. J. Med. 60, 501-580. 
GiL, D. M. 1982 Bacterial toxins: a table of lethal amounts. Microbiol. Revs. 46, 86-94.

GlenN, A. R. 1976 Production of extracellular proteins by bacteria. Ann. Rev. Microbiol. 30, $41-62$.

Grenney, W. J., Bella, D. A., \& Curl, H. C. 1973 A mathematical model of the nutrient dynamics of phytoplankton in a nitrate-limited environment. Biotechnol. Bioengng. 15, 331-358.

Heisnen, J. J. \& Roels, J. A. 1979 Application of balancing methods in modeling the penicillin fermentation. Biotechnol. Bioengng. 21, 2175-2201.

Herbert, D., Elsworth, R., \& Telling, R. C. 1956 The continuous culture of bacteria; a theoretical and experimental study. J. gen. Microbiol. 14, 601-622.

JoHNSON, M. J. 1967 Aerobic microbial growth at low oxygen concentrations. J. Bacteriol. 94, 101-108.

JoNEs, R. J. 1981 Vaccines and antisera against gram-negative bacilli. J. Hosp. Infect. 2, 105-111.

Kaushik, K. R., Gondo, S., \& Venkatasubramanian, K. 1979 Modeling of inducible enzyme biosynthesis in microbial cells. Ann. N.Y. Acad. Sci. 326, 57-72.

Koga, S., Burg, C. R., \& Humphrey, A. E. 1967 Computer simulation of fermentation systems. Appl. Microbiol. 15, 683-689.

MonOD, J. 1942 Recherches sur la Croissance des Cultures Bactériennes. Hermann, Paris.

NyBerg, K. \& Clarke, P. H. 1978 Glutamine synthetase activities of cultures of Pseudomonas aeruginosa grown in minimal media with histidine, nitrate or ammonium sulphate as the nitrogen source. J. gen. Microbiol. 107, 193-197.

Pamment, N. B., Hall, R. J., \& Barford, J. P. 1978 Mathematical modelling of lag phases in microbial growth. Biotechnol. Bioengng. 20, 349-382.

Priest, F. G. 1977 Extracellular enzyme synthesis in the genus Bacillus. Bacteriol. Revs. 41, 711-753.

PrurrT, B. A., JR. 1974 Infection caused by Pseudomonas aeruginosa species in patients with burns and other surgical patients. J. infect. Dis. 130, Suppl. S8-13.

Robinson, J. M., Keating, M. S., \& Sloan, G. L. 1980 The characteristics of extracellular protein secretion by Staphylococcus staphylolyticus. J. gen. Microbiol. 118, 529533.

Roels, J. A., \& Kossen, N. W. F. 1978 On the modelling of microbial metabolism. Prog. ind. Microbiol. 14, 95-203.

SHu, P. 1961 Mathematical models for the product accumulation in microbiological processes. J. biochem. microbiol. Technol. Engng 3, 95-109.

SipPOlA, M., \& MÄNTÄLÄ, P. 1981 Coproduction of several exoenzymes in Bacillus subtilis. FEMS microbiol. Letts. 10, 303-360.

Stiertz, D. D. \& Holder, I. A. 1975 Experimental studies of the pathogenesis of infections due to Pseudomonas aeruginosa: description of a burned mouse model. $J$. infect. Dis. 131, 688-691.

Szekely, J., Evans, J. W., \& Sohn, H. Y. 1976 Gas-Solid Reactions. New York: Academic Press.

van Dedem, G., \& Moo-Young, M. 1973 Cell growth and extracellular enzyme synthesis in fermentations. Biotechnol. Bioengng. 15, 419-439.

Verhoff, F. H., Sundaresan, K. R., \& TenNey, M. W. 1972 A mechanism of microbial cell growth. Biotechnol. Bioengng. 14, 411-436.

Wheatley, M. A., \& Moo-Young, M. 1977 Degradation of polysaccharides by endoand exoenzymes: dextran-dextranase model systems. Biotechnol. Bioengng. 19, 219433.

Williams, A. G., and WimpenNy, J. W. T. 1977 Exopolysaccharide production by Pseudomonas NCIB11264 grown in batch culture. J. gen. Microbiol. 102, 13-21.

Wrliams, F. M. 1967 A model of cell growth dynamics. J. theor. Biol. 15, 190-207. 